\title{
Mental health parity legislation: Anticipating costs and benefits
}

\author{
Arlene Smaldone ${ }^{1}$, Mary Cullen-Drill ${ }^{1}$ \\ Columbia University School of Nursing, New York, NY, USA
}

The Paul Wellstone and Pete Domenici Mental Health Parity and Addiction Equity Act was an important step in improving mental health coverage for many Americans. Prior to its implementation in January 2010, having health insurance did not assure equal access to Americans requiring mental health services. Often covered mental health benefits were more limited and restrictive compared to medical benefits and required greater consumer cost sharing when health insurance benefits were utilized. Key aspects of The Wellstone-Domenici Act are that it requires parity for mental health annual and lifetime out of pocket limits, addresses limits on outpatient visits or inpatient days, institutes parity for deductibles, coinsurance and co-payments, and eliminates arbitrary limits on days of coverage and frequency of treatments.

Unfortunately, the law has three major limitations. First, and most significant, is that an employer who offers health insurance as an employee benefit is not mandated by law to include mental health coverage as part of the insurance package. However, if mental health coverage is offered, it must be on par with medical coverage [1]. Although not the intent of the legislation, an employer could decide to drop medical coverage entirely to avoid the added cost of mental health parity and be operating within the scope of the law. Further, the law does not apply to out of pocket cost for prescription drugs. The latter is a critical omission for individuals with mental health disorders, as it is estimated that two thirds of out of pocket mental health spending is for prescription medications [2]. Second, the law does not apply to employers having less than 50 employees. As approximately 1 in 4 American workers are employed by small businesses, they are excluded from mental health parity protection [3]. Third, the Wellstone-Domenici Act provides no benefit to 49 million uninsured Americans.

Although far from ideal, the WellstoneDomenici Act was instrumental in shaping mental health benefits under the 2010 Patient Protection and Affordable Care Act (ACA) by establishing the core principle that coverage for mental and behavioral health be equal to that provided for other medical conditions [4]. The ACA builds on the Wellstone-Domenici Act by assuring mental health parity to more than 3.7 million currently uninsured Americans with severe mental illnesses as well as many others with less severe needs for mental health and addiction treatment services [5]. These individuals will gain access to health insurance, perhaps for the first time, as part of the 2014 health insurance expansion to approximately 30 million uninsured by either coverage under the publicly funded Medicaid program or subsidized purchase of health insurance coverage through state-based insurance exchanges. In addition, through ACA focus and support of primary care, including building the primary care workforce, better coordination and quality of care for those with mental health conditions can be anticipated. The Affordable Care Act was challenged by many states on the basis of its individual mandate for health insurance coverage. In June the US Supreme Court, in a 5 to 4 decision, upheld the broad intent of the law although the full uptake of Medicaid expansion by states remains unclear at this time. As implementation of the ACA moves forward, what economic ramifications of mental health parity should be expected? While the actual effect of mental health parity on cost and utilization of mental health services remains to be seen, experiences of both the 8.5 million federal employees and retirees insured by the Federal Employee Health Benefits (FEHB) Program and states that have recently initiated broad mental health parity expansions and evaluated its cost can provide insight for policymakers.

Initial evaluation of mental health parity by FEHB in 2001 did not demonstrate either increased cost or use of services during its first 2 years of implementation. However, the effect of parity on the sickest patients or those bearing the greatest financial burden of illness was unclear. Using longitudinal data for two years before and after par-
Corresponding author Arlene Smaldone ams130@mail.cumc. columbia.edu

\section{Disclosure}

The authors declare they have no fnancial competing interests 
ity implementation and simulation models, three subpopulations of mental health use were identifed: low, moderate and high spenders. Low spenders rarely sought mental health services whereas high spenders with chronic serious mental health conditions were frequent users of mental health services. Mental health parity did not affect service utilization for either group. However, the moderate spenders, primarily female, increased both use and mean spending after the introduction of parity [6].

In 2007 Oregon enacted comprehensive mental health parity that applied to all but self-insured plans. To examine the effect of parity on cost, mental health use and spending by commercially insured individuals was compared with that of individuals from selfinsured plans for two years before and after implementation of the law. No significant differences in mental health spending between groups were identified. These findings may be explained, in part, by the fact that parity affects only a relatively small group of individuals that require additional outpatient treatment as the greater cost of inpatient and prescription expenditures are not significantly affected by parity [7]. The findings of these studies are consistent with other prior evaluations of mental health parity suggesting that, on average, employer costs do not significantly increase following parity implementation and do not increase benefit utilization. However, as knowledge about the availability of health services is known to influence the decision to utilize services, consumer awareness of mental health parity is key.

Following implementation of its mental health parity legislation in 2007, a qualitative study was conducted in New York State to better understand employee knowledge and access to mental health benefits from a consumer perspective. Of the employees interviewed, all who were users of mental health services either for themselves or for a dependent child, most were unaware that their mental health benefits had changed. Lack of knowledge about benefits and poor communication from health care plans were identified as barriers to accessing mental health benefits. Of note, employees who had sought mental health services reported difficulty accessing high quality providers [8].

In 2014, many Americans will achieve access to health care including mental health parity through landmark ACA health reform. To assure access those newly enrolled must be knowledgeable regarding mental health parity benefits; therefore, health insurance plan communications to enrollees regarding scope of benefits must be monitored. As access to high quality mental health professionals is prerequisite to achieving true parity, assessment of adequacy of the current mental health workforce to meet this demand is needed. Finally, ongoing efforts to contain cost while promoting quality care should focus attention on the moderate spending group most likely to benefit from parity and coordination of care for users of mental health services.

\section{REFERENCES}

1. Research highlights: Are people with mental illness getting the help they need (2000). Retrieved December 20, 2009, from http://www.rand.org/pubs/research_briefs/RB4533/index1.html

2. Zuvekas SH, Meyerhoefer CD. State variations in the out-of-pocket spending burden for outpatient mental health treatment. Health Aff (Millwood) 2009; 28: 713-22. http://dx.doi.org/10.1377/hlthaff.28.3.713

3. Claxton G, DiJulio B, Finder B, et al. Employer health benefits. Menlo Park, CA: Kaiser Family Foundation and Health Research \& Education Trust, 2009

4. Barry CL, Huskamp HA, Goldman HH. A political history of federal mental health and addiction insurance parity. The Milbank Quarterly 2010; 88: 404-33. http://dx.doi.org/10.1111/j.1468-0009.2010.00605.x

5. Garfield RL, Zuvekas SH, Lave JR, et al. The impact of national health care reform on adults with severe mental disorders. Am J Psychiatry 2011; 168: 486-94. http://dx.doi.org/10.1176/appi.ajp.2010.10060792

6. Neelon B, O’Malley AJ, Normand ST. A Bayesian two-part latent class model for longitudinal medical expenditure data: Assessing the impact of mental health and substance abuse parity. Biometrics 2010; 67: 280-9. http://dx.doi. org/10.1111/j.1541-0420.2010.01439.x

7. McConnell KJ, Gast SH, Ridgely MS, et al. Behavioral health insurance parity: Does Oregon's experience presage the national experience with the mental health parity and addiction equity act? Am J Psychiatry 2012; 169: 31-8

8. Oziransky V, Jaksa A, Janopaul-Naylor E. Parity from the consumer perspective: Implications for federal implementation from New York's parity evaluation. Psychiatric Services 2011; 62: 344-6. http://dx.doi.org/10.1176/ appi.ps.62.4.344 